\title{
REAL-TIME DISPLACEMENT AND TILT ANALYSIS BY A SPECKLE TECHNIQUE USING $\mathrm{Bi}_{12} \mathrm{SiO}_{20}$ CRYSTALS
}

\author{
H.J. TIZIANI, K. LEONHARDT and J. KLENK \\ Institut für Technische Optik der Universität Stuttgart, \\ 7000 Stuttgart 80, Fed. Rep. Germany
}

Received 9 April 1980

Revised manuscript received 9 June 1980

Storage of speckle pattern in real time by means of $\mathrm{Bi}_{12} \mathrm{SiO}_{20}$-crystals will be reported. Applying the double exposure technique, deformations, displacements as well as tilts can be analysed. The novel speckle technique displays the Young'-interference fringes in quasi real time.

\section{Introduction}

Speckle photography is becoming an important method for deformation, displacement and tilt measurements. The technique will be even more attractive to the engineer when its application is in real time. Therefore, the developing process of speckle photography and the subsequent optical Fourier transformation of the photographs will be replaced.

Bismuth silicon oxyde $\mathrm{Bi}_{12} \mathrm{SiO}_{20}$, has electrical and electrooptical properties which make it attractive for dynamic holography and speckle applications. The basic principle for application of the crystal to holugraphic storage, double-exposure and time average interferometry of transparent or mirror like structures was reported $[1,2]$.

Upon readout of the hologram at the same wavelength the reconstructing light beam erases the stored information. Therefore, by the double-exposure technique, the second exposure partially erases the stored information. By the reconstruction, the resulting image with the interference fringes superimposed, can be stored by means of a video storage device or on film.

For real time holography and holographic interferometry the BSO crystal is biased with a transverse electric field $E_{0}$ in the 110 crystallographic direction. When the crystal is illuminated with spatially structured illumination in the $\overline{1} 10$ direction, a space-charge field is built up. A photoinduced space-charge density due to

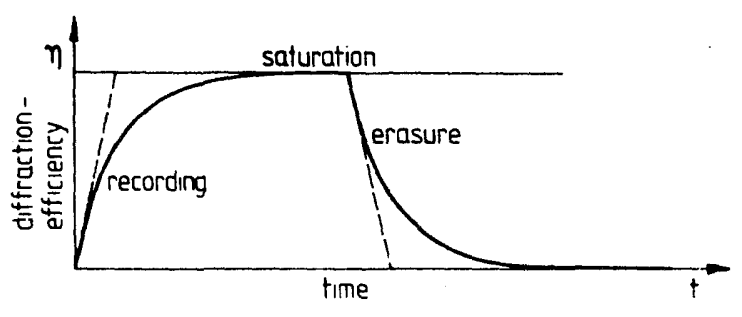

1:ig. 1. Diffraction efficiency by recording and erastre.

the difference between the distribution of trapped electrons and holes is generated [1]. The resultant spacecharge field changes the refractive index of the crystal via linear electrooptic effect. A phase volume hologram is created. Floading with uniform illumination leads to the erasure of the stored information by space-charge relaxation. Consequently, reading out with the recording wavelength is destructive. The diffraction efficiency $\eta$ as function of exposure time for one cycle of realtime holographic exposure is shown schematically in fig. 1 i 1$\}$.

\section{Storage of speckle patterns in BSO crystal}

Physical mechanisms for speckle pattern recording and erasure in BSO are drift and trapping of photoelectrons under illumination by a randorn light pattern field in the transverse electrooptic configuration. This gives 
rise to a space-charge field which modulates the refractive index via the linear electrooptic effect. Erasure is achieved by space-charge relaxation under uniform illumination. Applying the double exposure technique, two speckle patterns are recorded whereby the second is shifted relative to the first due to an object displacement or tilt between the two exposures. Depending on the optical configuration speckle patterns for inplane displacement or tilt analysis can be recorded [6]. The two speckle patterns are separated by at least one speckle dimension. therefore the intensity of the speckle patterns is considered to perforrn a spatial modulation of energy density in the crystal. The two stored speckle patterns are illuminated by a plane or converging wave. In the Fraunhofer plane Youngs' fringes are modulating the diffraction halo displayed by the speckle pattern. The fringe spacing is inversely proportional to the displacement.

For the crystals used the absorption coefficient was $\alpha_{1}=2 \mathrm{~cm}^{-1}$ for $\lambda_{1}=514 \mathrm{~nm}$ and $\alpha_{2}=0.28 \mathrm{~cm}^{-1}$ for $\lambda_{2}=633 \mathrm{~nm}$. The speckle patterns were therefore recorded with light of the wavelength $\lambda_{1}=514 \mathrm{~nm}$ and the Young"s fringes displayed with $\lambda_{2}=633 \mathrm{~nm}$. The crystal thickness $d$ was chosen to be $2.6 \mathrm{~mm}$.

The intensity distribution of the speckle pattern imaged onto the BSO leads to a space-charge field, $E_{\mathrm{sc}}$, by drift or diffusion of the photocarriers resulting in a refractive index variation. For speckles with dimensions $>$ I $u m$ the space-charge field is proportional to the electric field. $E_{0}$, applied [3]. The refractive index modulation at saturation, $\Delta n_{\mathrm{s}}$, can be written

$\Delta n_{\mathrm{s}}=n^{3} r E_{\mathrm{sc}} / 2$.

with $n=$ refractive index $(n \simeq 2.5$ for $\lambda=633 \mathrm{~nm}), r=$ effective electrooptic coefficient, $E_{\mathrm{sc}}=$ space-charge field amplitude which is derived from Poissons equation [1].

The variation of the refractive index due to the speckle pattern can be written as

$\Delta n=\Delta n_{s} C I^{\prime}\left(u^{\prime}\right) /\left\langle J^{\prime}\right\rangle$

where $I^{\prime}\left(u^{\prime}\right)$ is the intensity of the speckle pattern and $\left\langle I^{\prime}\right\rangle$ the mean intensity. $C$ is a constant taking into accouni the absorption in the crystal and reflection at the interface; it will be neglected. The recorded speckle pattern will now be illuminated with a wave of amplitude $A_{0}\left(u^{\prime}\right)$. The field amplitude considered to be immediately in front of the crystal will be written
$A^{\prime}\left(u^{\prime}\right)=A_{0}\left(u^{\prime}\right) \exp \left[\left(-\mathrm{i} 2 \pi / \lambda_{1}\right) d \Delta n\right]$,

$\lambda_{1}$ is the wavelength of recording and $d$ is the crystal thickness. The photoinduced phase shift is small; $d \Delta n \ll \lambda_{1}$ (it is of the order of $\lambda_{1} / 30$ ). The amplitude can therefore be written as

$A^{\prime}\left(u^{\prime}\right) \sim A_{0}\left(u^{\prime}\right)\left\{1-\mathrm{i} \frac{2 \pi}{\lambda_{1}} d \Delta n\right\}$,

where the terms on the order of $\Delta n^{2}$ and higher are neglected. In eq. (4) it is assumed that the length of the speckles corresponds at least to the crystal thickness $d$. It was found experimentally that this requirement is not necessary.

In the following section, a short theoretical analysis of image plane speckle pattern recording in BSO crystals will be given.

\section{Image plane speckle pattern recording in BSO crystals}

A short theoretical analysis neglecting the crystal activity, birefringence and Bragg effect will be given to explain the fringe pattern obtained. The intensity of the speckle pattern in the image plane can be written as follows [6]

$$
I\left(u^{\prime}\right)=\sum_{q} \sum_{p} \psi\left(x_{p}\right) \psi^{*}\left(x_{q}\right) \exp \left[\mathrm{i} 2 \pi u^{\prime}\left(x_{p}-x_{q}\right)\right]
$$

To simplify the writing the one-dimensicnal notation is used instead of a vector notation, according to fig. 2 .

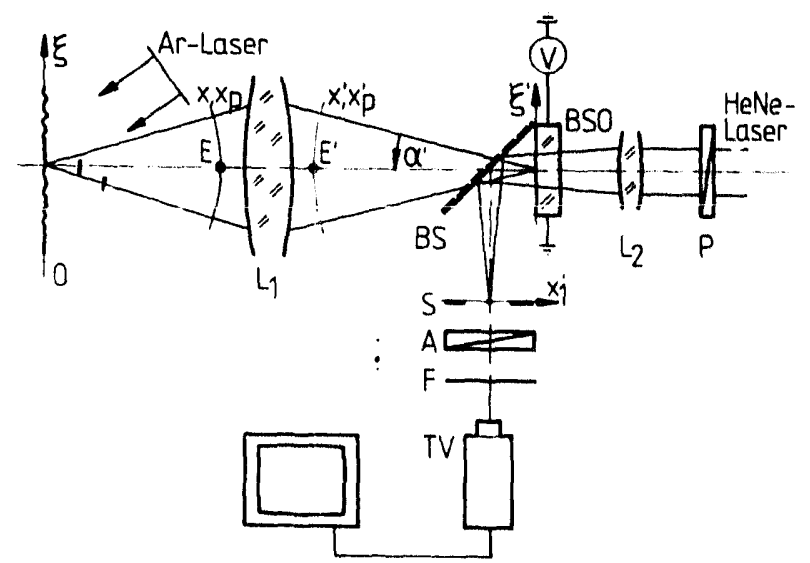

Fig. 2. Lxperimental arrangement for recording speckle palterns in the image plane and displaying Young's fringes. 
$u^{\prime}=$ reduced coordinate $; \quad u^{\prime}=\frac{n \sin \alpha^{\prime}}{\lambda} \xi^{\prime}$

$\xi^{\prime}=$ rectangular coordinate in the image plane

$\alpha^{\prime}=$ aperture angle in the image space

$x=x / h, x^{\prime}=x^{\prime} / h^{\prime}=$ reduced coordinates in the en-

trance and exit pupil

$h, h^{\prime}=$ pupil heights

$x_{p}, x_{q}=$ reduced coordinate in the entrance pupil plane $x_{p}^{\prime}, x_{q}=$ reduced coordinate in the exit pupile plane $\psi\left(x_{p}\right), \psi^{*}\left(x_{q}\right)$ describe the optically rough surface and represent the angular components of the light scattered by the optically rough surface.

In the double exposure technique two speckle patterns are recorded in the crystal. The first is stored at nearly saturation and the second, the shifted, is recorded incoherently because the speckle separation is greater than the speckle size. For the second exposure of the displayed speckle pattern the original speckle pattern will not be affected very much. The intensity of the two speckle patterns recorded in the crystal is according to eq. (5) using one-dimensional notation

$$
\begin{aligned}
& I\left(u^{\prime}, \Delta u^{\prime}\right)=C\left\{\sum_{q} \sum_{p} \psi\left(x_{p}\right) \psi^{*}\left(x_{q}\right)\right. \\
& \times \exp \left[\mathrm{i} 2 \pi u^{\prime}\left(x_{p}-x_{q}\right)\right]+\sum \sum \psi\left(x_{p}\right) \psi^{*}\left(x_{q}\right) \\
& \left.\quad \times \exp \left[\mathrm{i} 2 \pi\left(u^{\prime}+\Delta u^{\prime}\right)\left(x_{p}-x_{q}\right)\right]\right\}
\end{aligned}
$$

or by substituting $\boldsymbol{x}_{\boldsymbol{r}}$ for $\boldsymbol{x}_{\boldsymbol{p}}-\boldsymbol{x}_{\boldsymbol{q}}$

$$
\begin{aligned}
& I\left(u^{\prime}, \Delta u^{\prime}\right)=C\left\{\sum_{q} \sum_{r} \psi\left(x_{r}+x_{q}\right) \psi^{*}\left(x_{q}\right) \exp \left(\mathrm{i} 2 \pi u^{\prime} x_{r}\right)\right. \\
& +\sum_{q} \sum_{r} \psi\left(x_{r}+x_{q}\right) \psi^{*}\left(x_{q}\right) \\
& \left.\quad \times \exp \left[\mathrm{i} 2 \pi\left(u^{\prime}+\Delta u^{\prime}\right) x_{r}\right\rceil\right\} .
\end{aligned}
$$

The double exposed speckle pattern leads to a refractive index variation in the crystal volume.

\section{Displaying Young's fringes in the Fraunhofer plane}

Illuminating the crystal with a coherent wave of wavelength $\lambda_{2}$ and absorption coefficient $\alpha_{2}$ the wave- front emerging from the crystal can be written by neglecting Bragg volume effects in accordance with expressions (4)

$A^{\prime}\left(u^{\prime}\right)=A_{0}\left(u^{\prime}\right) C_{1}\left\{1-\frac{i 2 \pi}{\lambda} d \Delta n_{s} \frac{I\left(u^{\prime}, \Delta u^{\prime}\right)}{\langle I\rangle}\right\}$,

where $A_{0}\left(u^{\prime}\right)$ represents the incident wave amplitude and $C_{1}$ is a constant, taking also into account the small absorption in the crystal for $\lambda_{2}$ and the reflection at the interface. For the analysis $C_{1}$ will be neglected and a plane wave of constant amplitude $A_{0}$ is considered. The Fourier transform in the focal plane is

$$
\begin{aligned}
& a\left(x_{1}^{\prime}\right)=A_{0} \int_{\text {crystal }}\left\{1-\frac{\mathrm{i} 2 \pi}{\lambda_{1}} d \Delta n_{\mathrm{s}} \frac{I\left(u^{\prime}, \Delta u^{\prime}\right)}{\langle I\rangle}\right\} \\
& \quad \times \exp \left(\mathrm{i} 2 \pi x_{1}^{\prime} u^{\prime}\right) \mathrm{d} u^{\prime}
\end{aligned}
$$

and the intensity is

$$
\begin{aligned}
& \left|a\left(x_{1}^{\prime}\right)\right|^{2}=A_{0}^{2}\left\{\delta\left(x_{1}^{\prime}\right)+\left(\frac{2 \pi}{\lambda_{1}}-d \Delta n_{s}\right)^{2}\right. \\
& \left.\quad \times\left|\sum_{q} \frac{\psi\left(x_{1}^{\prime}+x_{q}\right) \psi^{*}\left(x_{q}\right)}{\langle I\rangle}\right|^{2}\left[1+\cos 2 \pi x_{1}^{\prime} \Delta u^{\prime}\right]\right\}^{(9)} .
\end{aligned}
$$

$\Delta n_{\mathrm{s}}=\left(n^{3} r E_{\mathrm{sc}} / 2\right)^{2}$

and $\Sigma_{q} \psi\left(x_{1}^{\prime}+x_{q}\right) \psi^{*}\left(x_{q}\right)$ is the autocorrelation of the speckle in the pupil of the image forming lens.

The square of the autocorrelation of the exit pupil of the image forming lens is modulated by Young's interference fringes.

The in-plane displacement in the object plane is given by

$\Delta \xi=f_{1}^{\prime} \lambda_{2} / M x_{c}^{\prime}$,

where $f_{1}^{\prime}=$ focal length or the distance from the crystal to the Fraunhofer-plane, depending on the experimental arrangement; $M=$ lateral magnification; $x_{c}^{\prime}=$ fringe spacing.

For the fully illuminated pupil of the image forming lens, the radius of the autocorrelation is

$r_{x_{1}^{\prime}}=2 f_{1}^{\prime} \sin \alpha^{\prime}$.

Consequently, no fringes van be observed for $\left|x_{1}^{\prime}\right|>$ $r_{x_{1}^{\prime}}$. 
in the experiment it was found, however, that the fringe envelope is very often smaller. This may be due Wo the orystal thickness (Bragg effect) [8]. Furthermore, crystal activity and birefringence may influence the envelope of the Young's fringes.

A similar theory can be developed for tilt analysis by recording the speckle pattern in the Fourier transform planc of lens $L_{1}$ in fig. 2 [6] or in the image of the light source. In addition, the simplified theory can be extended to two dimensions.

\section{Some experimental results}

A Iypical experimental set-up for real time speckle patterr recording is shown in fig. 2 for displacement analysis. The optically rough surface was illuminated with an argon laser $\left(\lambda_{1}=514 \mathrm{~nm}\right)$. The low aperture lens $(\mathrm{NA}=0.06$ ) formed the image in the crystal. The power on the crystal to write the speckle pattern was

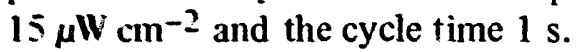

The BSO crystal was illuminated with a HeNe laser $(1 \mathrm{~mW}) \lambda_{2}=633 \mathrm{~nm}$. The Young's fringes were displayed in the Fraunhofer plane where a stop was placed to elininate the undiffracted light; the fringes were observed on a TV screen. A dichroic beamsplitter BS retlocis $\lambda_{2}$ and the analysator $A$ reduces stray light $\left[-D_{0}\right.$. Fur ther details of the experimental arrangements Will be described in a later paper.

In fig. 3 fringe patterns of displacements (fig. 3a) and tilts (fig. $3 \mathrm{~b}$ ) are observed. The object was a diffuse reflecting rough surface. The displacements were applied perpendicular to the applied field $E_{0}(4.8 \mathrm{kV})$ and tilts in a direction perpendicular to the first $\left(f_{1}^{\prime}=\right.$ $90 \mathrm{~mm}$ ).

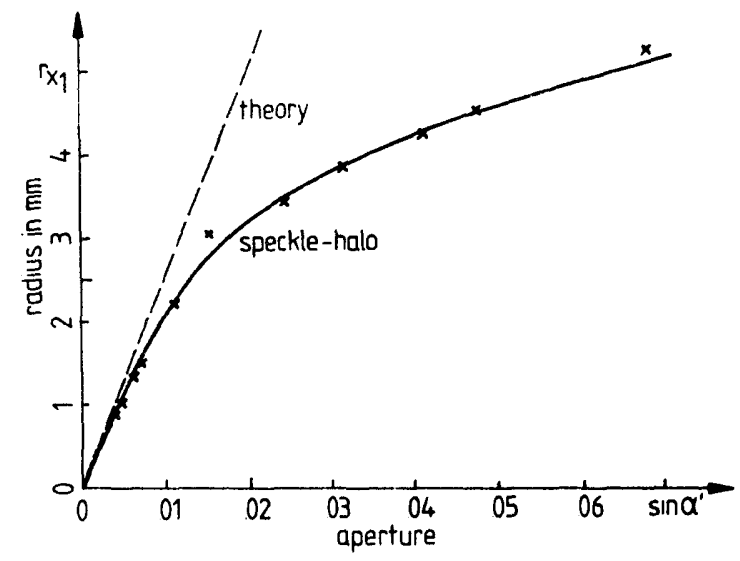

Fig. 4. Speckle halo plotted against the numerical aperture obtained with an arrangement corresponding to fig. 2 .

In our analysis, the Bragg volume effect as well as crystal activity and birefringence were neglected. The experiments showed, however, that they can limit the speckle halo as shown in fig. 4 where the radius of the speckle halo $r_{x_{1}^{\prime}}$ is plotted against the numerical aperture of lers $L_{1}$ in the image space. For small $\alpha^{\prime}$ the autocorrelation of the pupil is limiting the halo as pre. dicted. For larger $\alpha^{\prime}$. however, the Bragg volume effect and the birefringence are probably limiting the field in which Young's fringes are observed.

\section{Conclusions}

Using BSO crystals in the transverse electroopticmode a real time operation of "speckle photography" techniques is achieved with unlimited recycling. The fringe analysis can be obtained directly using TV tech.
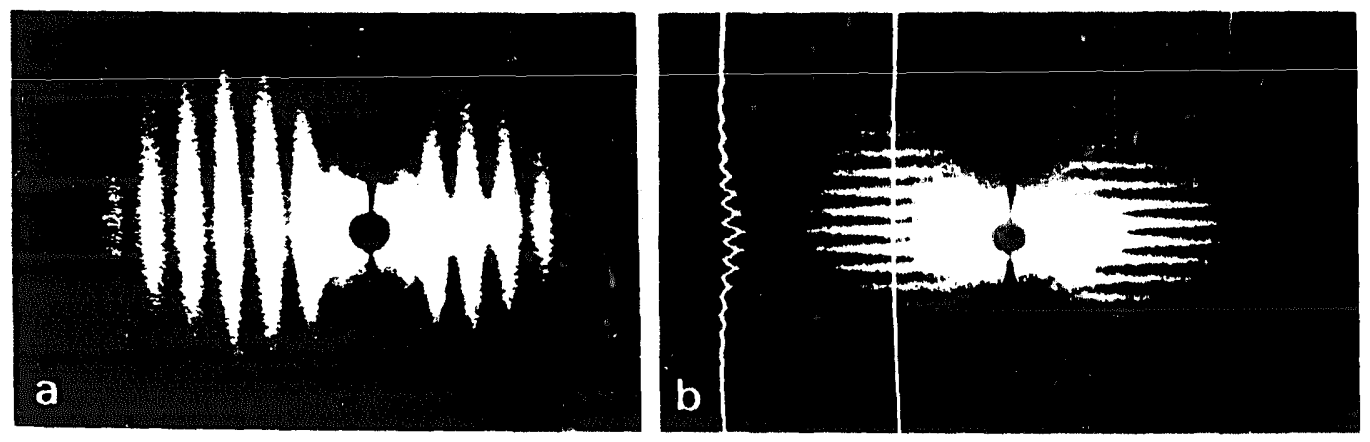

Fig. 3. Young's fringes obtained by a) an inplanc displacement of $100 \mu \mathrm{m}$, b) a tilt of 25 sec of are. 
niques, as indicated in fig. $3 b$, where the fringe pattern is scanned directly. Further studies and applications are in preparation and will be reported.

We would like to thank J.P. Huignard and his colleagues for very helpful advice in handling the BSO crystals and the DFG for the financial support.

\section{References}

[1] J.P. Huignard and F. Micheron, Appl. Phys. Lett. 29 (1976) 591.
[2] J.P. Huignard, J.P. Herriau and T. Valentin, App!. Optics 16 (1977) 2796;

J.P. Huignard and J.P. Herriau, Appl. Optics 16 (1977) 1897.

[3] J.P. Huignard, J.P. Herriau, G. Rivet and P. Günter, Optics Letters 5 (1980) 102.

[4] J.P. Herriau, J.P. Huignard and P. Auborg, Appl. Optics 17 (1978) 1851.

[5] M.P. Petrov, S.V. Miridonov, S.I. Stepanov, and V.V. Kulikov, Optics Comm. 31 (1979) 301.

[6] H.J. Tiziani, in: Speckle metrology, ed. R.K. Erf (Academii Press, 1978) p. 73.

[7] A.E. Ennos, in: Laser speckle and related phenomena, ed. J.C. Dainty (Springer, Berlin, 1975) p. 237.

[8] H. Kogelnik, Bell Syst. Techn. J. 48 (1969) 2909. 